\title{
IST PEER-REVIEW NOCH DIE RICHTIGE EVALUATIONSMETHODE?
}

\author{
THOMAS SPITZLEY \\ DOI: 10.22163/fteval.2019.458
}

$\mathrm{P}$ eer-Review wird im universitären Kontext u.a. a) für die Begutachtung von Forschungsvorhaben oder Publikationen, b) im Rahmen von Auswahlverfahren (z.B. bei Berufungen) und c) bei externen Evaluierungen von Einrichtungen oder Programmen (z.B. Hochschulen, Fakultäten, Instituten oder Studiengängen) eingesetzt. In den Fällen a) und b) kann man mit guten Gründen dafür argumentieren, dass Peer-Review die richtige, wenn nicht sogar die einzig adäquate Evaluationsmethode ist. Dieser Beitrag beschränkt sich auf die Betrachtung der in c) genannten Fälle, genauer: auf die Evaluation von Studiengängen und Studienbedingungen, und hier ist eine komplexere Antwort zu geben.

Die Peer-Review-Evaluation von Studiengängen wurde im Zuge des Bolognaprozesses flächendeckend im Rahmen von Akkreditierungsverfahren eingeführt. Durch die Überprüfung, ob die damals geschaffenen Standards und Leitlinien für die Qualitätssicherung im Europäischen Hochschulraum (ESG) ${ }^{1}$ eingehalten werden, sollten die Studiengänge transparenter und vergleichbarer werden. Grundsätzlich war Peer-Review in diesem Kontext Bestandteil eines dreistufigen Verfahrens:

(1) interne Evaluation in Form eines Selbstberichts,

(2) externe Evaluation in Form einer u.U. datengestützten Begutachtung durch Peers,

(3) Umsetzung von Follow-up Maßnahmen (Auflagen und Empfehlungen).

Die ESG setzen Peers mit Expert*innen gleich. Typischerweise sind Expert*innen allerdings denjenigen, von denen sie beauftragt werden, fachlich überlegen. ${ }^{2}$ Peers haben dagegen grundsätzlich dieselbe Fachkompetenz wie diejenigen, die sie beurteilen bzw. beraten sollen. Das heißt jedoch nicht, dass Peers über eine geringere Kompetenz verfügen als Expert*innen, sondern im Gegenteil, dass auch den Begutachteten ein Expert*innenstatus zukommt.

Aus dem hier relevanten Gegenstand der Evaluation, nämlich Studiengänge und Studienbedingungen, ergibt sich, dass zu den Peers nicht nur Wissenschaftler*innen mit entsprechender Lehrerfahrung gehören dürfen, sondern dass auch Studierende dazugehören müssen.

Wenn man ernst nimmt, dass die Peers keine höhere Kompetenz haben als die Begutachteten, dann kann den Urteilen der Peers nicht automatisch ein größeres Gewicht zukommen. Insofern ist es folgerichtig, dass sich das Verständnis von Peers als "Richter*innen“, die ein in der
Regel abschließendes Urteil fällen, hin zu "critical friends" gewandelt hat, die den Betroffenen auf Augenhöhe Ratschläge geben. Peers als critical friends

a) treten den Begutachteten grundsätzlich wohlwollend und fair gegenüber,

b) abstrahieren von ihren eigenen Vorstellungen und Präferenzen,

c) berücksichtigen die Interessen der Begutachteten und nicht die eigenen Interessen,

d) beziehen sich nicht schlicht auf ihren eigenen Erfahrungshintergrund als mutmaßliche best practice, sondern orientieren sich in ihrer Urteilsbildung an der konkreten Situation, in der sich die Begutachteten befinden, und

e) richten ihre Empfehlungen stets auf Qualitätsverbesserung aus.

Strittig ist, ob und, wenn ja, in welchem Maße Peers generell nicht nur über fachwissenschaftliche Kompetenz verfügen, sondern auch hinreichend sachkundig in Bezug auf Evaluationen sein müssen. So plädiert Gutknecht-Gmeiner beispielsweise dafür, dass u.a. „bei der Evaluation der Lehre [...] [e]inschlägige fachliche Expertise [...] hier ausgewiesenen Hochschuldidaktiker*innen [...] zuzuschreiben [ist]; diese wären also [...] als ,Sachverständige' geeignet, nicht jedoch Fachkolleg*innen aus der evaluierten Disziplin, d.h. Peers, oder Vertreter*innen von Stakeholdern". ${ }^{3}$ Am weitesten verbreitet ist die Auffassung, dass die Peers zumindest über Grundkenntnisse und -kompetenzen im Bereich Evaluationen verfügen müssen, und daher werden den Gutachter*innen von den Akkreditierungsagenturen normalerweise entsprechende Informationsmaterialien bzw. Schulungen angeboten. Folgt man dieser Auffassung, benötigen Peers bei der Evaluation von Studiengängen also eine Doppelkompetenz, nämlich sowohl eine wissenschaftlich-fachliche als auch eine im Bereich Evaluationen, und das bedeutet, dass Peers zumindest in der zweiten Hinsicht in der Regel über eine größere Expertise verfügen als diejenigen, die sie beurteilen bzw. beraten sollen (s.o.).

Im Idealfall findet durch flächendeckende Peer-Review-Evaluationen ein umfassender Austausch guter Ideen für die Gestaltung von Studiengängen statt, der dazu führt, dass deren Qualität überall steigt, nicht nur an der gerade evaluierten Einrichtung, dass sowohl die Evaluierten als auch die Peers von dem Austausch profitieren und dass unter den Beteiligten sogar längerfristige Kooperationen oder Netzwerke entstehen. In 
der Realität gibt es allerdings deutliche Zweifel an der Wirksamkeit von Peer-Review-Evaluationen.

Schon diese kurze Skizze lässt aber deutlich werden, dass hohe Anforderungen erfüllt sein müssen, damit Peer-Review-Evaluationen erfolgreich sein können. Daneben müssen für jede Evaluation Peers gefunden werden, die auch fachlich einschlägig sind, doch das ist eine erhebliche Herausforderung: ${ }^{4}$ Die Zahl der Bachelor- und Masterstudiengänge steigt von Jahr zu Jahr; allein in Deutschland wurden im WS 2018/19 knapp 18.000 Bachelor- und Masterstudiengänge angeboten, ${ }^{5}$ die regelmäßig akkreditiert werden müssen. Gleichzeitig sinkt angesichts der kontinuierlich steigenden administrativen Aufgaben, die Wissenschaftler*innen zu erfüllen haben, die Bereitschaft zur Mitwirkung an Evaluationen. Intensive Gutachtertätigkeit kann zwar auch zu Reputationssteigerung führen, doch nicht in demselben Maße, wie es bei herausragenden wissenschaftlichen Leistungen der Fall wäre, die in derselben Zeit erzielt werden könnten, und die von den Peers aufzubringende Zeit wird auch nicht durch die in der Regel gezahlten Aufwandspauschalen kompensiert. ${ }^{6}$

Auch auf der Seite der Betroffenen kommen noch weitere Schwierigkeiten hinzu: Die Betroffenen müssen sich an dem Verfahren konstruktiv beteiligen, das setzt die Akzeptanz des Verfahrens voraus, und die hängt nicht zuletzt von den möglichen Konsequenzen der Evaluation ab. Für die Betroffenen muss der u.a. für die Erstellung des Selbstberichts zu betreibende Aufwand in einer vernünftigen Relation zum Ertrag des Verfahrens stehen, aber in der Regel ist zu Beginn des Verfahrens weder der Ertrag klar, noch sind die Konsequenzen absehbar. Darüber hinaus gilt das im Zusammenhang mit den Peers genannte Zeitproblem für die Betroffenen in gleichem Maße.

Schließlich ist noch eine weitere Schwierigkeit zu erwähnen, die mit Peer-Review-Evaluationen einhergeht: Aufgrund der Beteiligung externer Peers und der von ihnen mutmaßlich ausgeübten externen Kontrolle wird häufig eine Einschränkung der Autonomie der Hochschule im Allgemeinen und der an der Lehre beteiligten Wissenschaftler*innen im Besonderen konstatiert.

Die genannten Probleme mit Peer-Review-Evaluationen betreffen somit im Wesentlichen drei Dimensionen: zeitliche Belastung, Akzeptanz und Autonomie. Um sie zu lösen, muss die erforderliche zeitliche Belastung reduziert, die Akzeptanz erhöht und die Autonomie gestärkt werden. Eine Möglichkeit, um dies zu erreichen, soll an dem folgenden Beispiel kurz demonstriert werden.

In Deutschland wurde den Hochschulen mit der Möglichkeit zur Systemakkreditierung (in Österreich Audit) die Gelegenheit gegeben, die gesamten Qualitätsmanagementprozesse, und eben auch die Peer-ReviewEvaluationen, deutlich bedarfsgerechter zu gestalten. In Vorbereitung auf ihre Systemakkreditierung hat die Universität Duisburg-Essen vor wenigen Jahren ihr etabliertes Verfahren regelmäßiger, sechsjährlicher institutioneller Evaluationen (mit Peer-Review) der Fakultäten so modifiziert, dass zusätzlich zu den bisherigen Betrachtungsgegenständen ein stärkerer Fokus auf die Studiengänge gelegt wird. Die regelmäßigen Peer-Review-Evaluationen, die früher als Programm(re)akkreditierungen stattfanden, sind dafür entfallen. So konnte die große zeitliche Belastung der Fakultäten deutlich reduziert werden. Im Gegenzug wurden die Instrumente der Selbstreflexion durch jährlich stattfindende Qualitätsgespräche zu den einzelnen Lehreinheiten und sechsjährlich stattfindende vertiefte interne Betrachtungen der einzelnen Studiengänge verfeinert. Dadurch, aber auch durch einen deutlicheren Hinweis darauf, dass die Fakultäten sich im Zuge der institutionellen Evaluation durch das Erstellen eines Selbstberichts zunächst selbst evaluieren, wird die Verantwortung der betroffenen Wissenschaftler*innen gestärkt und ihre Autonomie erhöht. Statt Peer-Review-Evaluationen im Rahmen von regelmäßigen Verfahren zur Programm(re)akkreditierung gibt es an der Universität Duisburg-Essen Peer-Review mit Bezug auf Studiengänge nun nur anlassbezogen, d.h. dann, wenn die für die Einrichtung und Durchführung von Studiengängen Verantwortlichen einen Rat von kompetenten critical friends brauchen. Auch dadurch wird die Autonomie der beteiligten Wissenschaftler*innen, aber auch die Autonomie der Hochschule als ganzer erhöht. Dazu trägt auch bei, dass bei der Durchführung aller Evaluationen hochschulintern professionell auf Qualitätssicherung geachtet wird. Dadurch verliert nämlich die oben erwähnte Doppelkompetenz der Peers an Bedeutung, und es kommt bei Peer-Review Evaluationen weitestgehend nur auf die wissenschaftlich-fachliche Expertise der externen Peers an, sodass die internen Wissenschaftler*innen in der Tat mit innen auf Augenhöhe sind.

Mit der so gestärkten Autonomie der Universität geht allerdings eine hohe Erwartung einher: Es wird vorausgesetzt, dass die Verantwortlichen nicht nur die Gelegenheiten erkennen, bei denen ihnen ein solcher Rat helfen würde, sondern dann auch bereit sind, ihn einzuholen. Ob die für die Einrichtung und Durchführung von Studiengängen Verantwortlichen sich bei einer derartigen stärkeren Selbststeuerung auch entsprechend verantwortlich verhalten, wird im Rahmen der System-Reakkreditierung zu überprüfen sein.? Außerdem bleibt abzuwarten, ob die genannten Maßnahmen den beabsichtigten Effekt hatten und die Akzeptanz der Betroffenen für Qualitätssicherung im Allgemeinen und Peer-Review Evaluationen im Besonderen erhöhen.

Fazit: Auch wenn es künftig aus guten Gründen keine flächendeckenden Peer-Review Evaluationen mehr geben wird, wie es in den vergangenen Jahrzehnten üblich war, wird man trotz aller Schwierigkeiten ebenfalls aus guten Gründen - nicht auf die Einschätzung externer Peers verzichten können. ${ }^{8}$

$4 \quad$ Für Österreichische Audit Verfahren hat die A0 Austria aufgezeigt, wie je nach Agentur die Zusammensetzung der Peers z.B. hinsichtlich ihrer Erfahrung in der Studienganggestaltung, ihrer Berufspraxis und der internationalen Dimension voneinander abweichen (vgl. F. Blüml et al., Auditverfahren in Österreich, hrsgg. von A0 Austria, Wien 2019, S.17-19).

$5 \quad$ Vgl. HRK (Hrsg.), Statistische Daten zu Studienangeboten an Hochschulen in Deutschland. Studiengänge, Studierende, Absolventinnen und Absolventen Wintersemester 2018/2019, Statistiken zur Hochschulpolitik 1/2018, S.9 (www.hrk.de/fileadmin/redaktion/hrk/02-Dokumente/02-06-Hochschulsystem/ Statistik/HRK_Statistik_BA_MA_UEbrige_WiSe_2018_19.pdf; abgerufen am 6.7.2019)

6 Vgl. Neidhardt, F., „Selbststeuerung der Wissenschaft durch Peer-Review-Verfahren“, S.269f., in: D. Simon et al. (Hrsgg.), Handbuch Wissenschaftspolitik, Springer, Wiesbaden 2016, S.261-277.

$7 \quad$ Mit dieser Vorgehensweise folgt die Universität der Empfehlung des Wissenschaftsrates: „Die Hochschulen sollten das Oualitätsmanagement von Lehre und Forschung verstärkt als Kernelement ihrer Autonomie verstehen und dafür effiziente, wissenschaftsadäquate Verfahren entwickeln." (Wissenschaftsrat, Empfehlungen zur künftigen Rolle der Universitäten im Wissenschaftssystem, Köln 2006, S.70) Für hilfreiche Anregungen und Unterstützung danke ich Martina Rendel. 


\section{QUELLEN}

Gutknecht-Gmeiner, M., „Experten, Gutachterinnen oder Dilettanten: Welche Evaluationskompetenz und Schulung benötigen Peers?, S.237, in: Zeitschrift für Evaluation 12, 2013, S.235-256.

Neidhardt, F., "Selbststeuerung der Wissenschaft durch Peer-ReviewVerfahren", S.269f., in: D. Simon et al. (Hrsgg.), Handbuch Wissenschaftspolitik, Springer, Wiesbaden 2016, S.261-277.

\section{AUTOR}

PROF. DR. THOMAS SPITZLEY

Prorektor für Entwicklungs- und Ressourcenplanung

Universität Duisburg-Essen

Universitätsstr. 12, 45117 Essen

E: thomas.spitzley@uni-due.de 